\title{
STIMULUS-DRIVEN SELECTION OF ROUTES TO IMITATION
}

\author{
Clare Press*, \& Cecilia Heyes \\ * Corresponding author \\ Department of Psychology \\ University College London \\ 26 Bedford Way, London. WC1H 0AP \\ United Kingdom
}

Phone: 44 (0)20 76795379

Fax: 44 (0)20 74364276

Email: c.press@ucl.ac.uk

URL: http://www.psychol.ucl.ac.uk/celia.heyes/netintro.htm 


\section{ABSTRACT}

Several models have proposed that an action can be imitated via one of two routes: a direct visuospatial route, which can in principle mediate imitation of both meaningful and meaningless actions, and an indirect semantic route, which can be used only for meaningful actions. The present study investigated whether selection between the direct and indirect routes is strategic or stimulus-driven. It has previously been shown, using accuracy measures, that imitation of meaningful actions is superior to imitation of meaningless actions when the two action types are presented in separate blocks, and that the advantage of meaningful over meaningless items is smaller or absent when they are presented in mixed blocks. We first replicated this finding using an automated, reaction time (RT) procedure. We then examined latency to imitate meaningful and meaningless actions in the mixed condition as a function of the action type presented in the previous trial, and in relation to the number of previous test trials. These analyses showed that 1 ) for both action types, performance was worse immediately after meaningless than after meaningful trials, and 2) even at the beginning of the mixed condition, responding to meaningful actions was no faster than responding to meaningless items. These results suggest that the properties of the action stimulus play a substantial role in determining whether imitation is mediated by the direct or the indirect route, and that effects of block composition on imitation need not be generated through strategic switching between routes.

Keywords: Imitation, semantics, working memory, two-route model, associative sequence learning. 


\section{INTRODUCTION}

It has been proposed that action imitation can be accomplished via two routes (e.g. Rothi, Ochipa, \& Heilman, 1991; Rumiati \& Tessari, 2002; Tessari \& Rumiati, 2004). This theory proposes that unfamiliar or 'meaningless' (ML) actions are imitated by mapping an observed action directly onto an executed action, according to its visuospatial properties. In principle, familiar or 'meaningful' (MF) actions can also be imitated via this direct visuospatial route, but in addition, they can be imitated via an indirect semantic route. This semantic route involves an additional stage of processing; between visual analysis and preparation of motor output, a semantic representation of the action is retrieved from long term memory. Neuroimaging and neuropsychological research have provided support for the existence of two routes of imitation. In a positron emission tomography (PET) study, it was found that observation of MF actions activated the left hemisphere in frontal and temporal regions, relative to observation of ML actions, whereas observation of ML actions activated the right parieto-occipital junction, relative to observation of MF actions (Decety et al., 1997; see also Rumiati et al., 2005). In addition, Tessari, Canessa, Ukmar, \& Rumiati (2007) found that apraxic patients who were better able to imitate MF than ML actions had lesions in the superior temporal lobe and the ventral portion of the angular gyrus, whereas patients who were better able to imitate ML than MF actions had lesions affecting the hippocampus and dorsal angular gyrus.

However, in a behavioural study with neurologically healthy participants, Tessari \& Rumiati (2004) found evidence suggesting that MF and ML actions are not always imitated via distinct routes. Imitation of ML actions was less accurate than imitation 
of MF actions when the two action types were presented in separate blocks (blocked condition), but there was no detectable difference in the accuracy of imitating MF and ML actions when they were presented in random order within the same blocks (mixed condition). Similar results were obtained when testing patients with apraxia (Tessari et al. 2007). On the basis of these findings, Tessari \& Rumiati (2004) proposed that ML actions must always be imitated via the direct route, because ML actions are not represented in semantic long-term memory, but the route for imitation of MF actions can be selected strategically, according to which is likely to be more efficient. The indirect route places fewer demands on working memory than the direct route because semantic long-term memory represents each action as a unit, whereas the direct route requires that the action be decomposed into chunks that are each held in working memory. Therefore, the direct route will be selected when MF actions are presented in the blocked condition. However, in the mixed condition, use of distinct routes for the two action types incurs switch costs, and these switch costs outweigh the advantages of using the indirect route for MF actions. Therefore, in the mixed condition, it is more efficient to select the direct route for both action types. Given that their participants were not instructed about the composition of the block, Tessari \& Rumiati (Rumiati et al., 2005) proposed that, in the mixed condition, participants switched between routes for a few trials, discovered from this experience that switching was inefficient, and then strategically selected the direct route for use in all remaining trials.

Thus, the lack of an observable performance difference between imitation of MF and ML actions under mixed conditions may be due to strategic selection of the direct route for both action types. However, this pattern of results is also compatible with 
stimulus-driven route selection, in which the perception of a MF or familiar action automatically activates the indirect route, whereas perception of a ML or unfamiliar action automatically activates the direct route. This alternative hypothesis implies that, even under mixed conditions, imitation of MF actions is mediated by the indirect route and imitation of ML items is mediated by the direct route. However, the advantage of MF over ML items is lost under mixed conditions because processing of a ML item, via the direct route, makes heavy demands on working memory and thereby interferes with processing of any item that follows it in the list. When presentation is blocked, this carry-over cost is borne solely by other ML items, enhancing the advantage of MF over ML items. However, when MF and ML items are presented in random order, the carry-over cost affects the imitation of ML actions in half of the trials, and of MF actions in half of the trials. Therefore, it erodes the advantage of MF over ML items.

Therefore, the strategic selection and stimulus selection hypotheses both assume that there are two routes of imitation - a direct visuospatial route, and an indirect semantic route - and that ML, or unfamiliar, actions are always imitated via the direct route. They differ with respect to the processing of MF actions. The strategic selection hypothesis suggests that the indirect route is selected for MF actions when they are presented alone (blocked conditions), but, because of costs associated with switching between routes, the direct route is selected for MF actions when they are intermixed with ML actions (mixed conditions). In contrast, the stimulus selection hypothesis suggests that MF actions are imitated via the indirect route under both blocked and mixed conditions, and that they lose their advantage over ML actions under mixed 
conditions because the working memory costs associated with the direct route have a detrimental effect on performance in any trial that follows imitation of a ML action.

We tested the stimulus selection hypothesis against the strategic selection hypothesis by examining the effects of block composition (blocked versus mixed) on the imitation of MF and ML actions in a sample of neurologically healthy participants. There is a risk of ceiling effects when imitation accuracy is measured in neurologically healthy participants. Therefore, to enhance measurement sensitivity, we used a reaction time (RT) index of imitative performance.

The strategic selection and stimulus selection hypotheses both predict that MF actions will be imitated faster than ML actions in blocked conditions, and that this advantage for MF actions will be greater than that in mixed conditions. However, the hypotheses make different predictions about the pattern of data in the mixed condition. First, the stimulus selection hypothesis predicts that when either MF or ML actions are preceded by a MF action, they will be imitated faster than when they are preceded by a ML action. In contrast, the strategic selection hypothesis predicts no difference in RT according to the action type that precedes an action, because all actions are imitated via the direct route and therefore, all place equal demands on working memory. Second, the strategic selection hypothesis predicts faster responding to MF than ML items early in testing under mixed conditions, during the period when participants are switching between routes and learning that that this is not efficient, and subsequent loss of this advantage when they resort to using the direct route for both action types. In contrast, the stimulus selection hypothesis predicts no such change in the course of mixed blocks, because it assumes that 
distinct routes are used throughout for the imitation of MF and ML actions.

Therefore, the initial purpose of this study was to replicate, using an RT measure, the finding that imitation of MF actions is superior to that of ML actions under blocked conditions, and that difference is reduced under mixed conditions (Tessari \& Rumiati, 2004). We then investigated the processes underlying these effects by analysing performance for MF and ML action types in the mixed condition according to 1) whether an action item was preceded by a MF or a ML action, and 2) the stage of testing in the mixed condition.

\section{METHOD}

\section{Participants}

Twenty-four consenting, healthy volunteers with an average age of 21.9 years, 10 male, took part in the experiment, and were paid a small honorarium for their participation. All were right-handed, had normal or corrected-to-normal vision, and were naïve with respect to the purpose of the experiment. The study was approved by the University College London ethics committee, and performed in accordance with the ethical standards laid down in the 1964 Declaration of Helsinki.

\section{Stimuli}

All stimuli were presented on a computer screen $(75 \mathrm{~Hz}, 400 \mathrm{~mm}, 96 \mathrm{DPI})$, in colour on a black background, and viewing was unrestrained at a distance of approximately 1.5m. Each imperative stimulus was a hand and arm action performed by a male 
model with his left hand. The stimulus display subtended approximately $8.9^{\circ}$ of visual angle horizontally and $6.6^{\circ}$ vertically. There were 20 pantomimes of MF actions and $20 \mathrm{ML}$ actions, and the ML actions were constructed by performing MF actions with a different spatial relationship between the hand and arm, and the trunk. For example, the MF action of drinking consisted of bringing the hand from the front of the torso to the mouth, with the hand in a ' $\mathrm{C}$ ' formation, at first in the horizontal plane, and then rotating to the vertical plane. The ML version consisted of bringing the hand to the forehead rather than the mouth, but all other components of the action were identical. The videos were those used by Tessari \& Rumiati (2004), and the action items are listed in appendix A.

\section{Procedure}

Participants were tested individually in a well lit room. They were required to stand, and to hold down a large response button $(9.5 \mathrm{~cm}$ diameter) with their right hand. The button was positioned at hip height, and approximately $20 \mathrm{~cm}$ in front of the participant's body. They were instructed to release the response button in order to imitate the observed action with their right hand, and to ensure that they returned their hand to the button before a beep sounded, signalling the onset of the next trial. Given that stimulus actions were performed with the model's left hand, the executed actions matched the observed actions spatially, but not anatomically.

There were four blocks: one block contained only MF actions (blocked MF), one block contained only ML actions (blocked ML), and two blocks contained MF and ML actions (mixed). Whether participants undertook the blocked or mixed condition 
first was counterbalanced, as was the order of blocked MF and blocked ML conditions.

All trials began with presentation of the stimulus action, which was of $1500 \mathrm{~ms}$ duration. This was followed by a black screen, which was accompanied 1000ms later by a beep of 250ms duration, after which the next stimulus action was presented. Each block presented, in random order, 80 stimulus trials. In the blocked condition, a block consisted of four repetitions of each of 20 action stimuli. In the mixed condition, a block consisted of two repetitions of each of 40 action stimuli. Before testing commenced, participants completed 12 practice trials consisting of six MF actions and six ML actions. These actions were not presented in the test blocks.

In addition to RTs, the executed actions were scored for similarity to the observed actions on a 5-point scale, where 1 was the least similar and 5 was the most similar. An action rated as ' 1 ' bore no similarity at all to the observed action, i.e., the spatial trajectory of the hand and arm matched the observed action no more than any of the other actions in the stimulus set. An action rated as ' 5 ' was a perfect match, i.e., the positions and trajectories of the participant's hand and arm were indistinguishable from those of the model. Scores of 2, 3, and 4, were given to actions which fell between these levels of similarity, with approximately equal intervals between each of the points on the scale. The scorer was naïve to the hypotheses and predictions under investigation. 


\section{RESULTS}

Mean RT to imitate MF and ML actions, in the blocked and mixed conditions, was calculated for each participant. Trials rated as ' 1 ' for similarity $(0.7 \%)$, RTs under 150ms (3.2\%), and trials in which participants had not returned their hand to the button before the start of the trial (5.7\%), were excluded from the RT analysis. (The same results as those reported below were obtained when trials rated '1' and '2' for similarity were excluded from the analysis).

The RT data (Figure 1, columns) were subjected to ANOVA in which block type (blocked versus mixed) and action type (MF versus ML) were within-subjects variables. This analysis indicated a block type $\mathrm{x}$ action type interaction $(\mathrm{F}(1,23)=$ 10.2, $\mathrm{p}<0.005)$. Simple effects analyses indicated faster RTs to imitate MF than ML actions in the blocked condition $(F(1,23)=7.6, \mathrm{p}<0.02)$, but not in the mixed condition $(F(1,23)=2.5, p=0.1)$. Analysis of the similarity data (Figure 1, lines) indicated that the foregoing effects on RT were not due to a speed-accuracy trade-off. In the blocked condition, MF actions were imitated more accurately than ML actions $(F(1,23)=20.0, p<0.001)$, and in the mixed condition there was a lesser advantage of MF over ML items (block type x action type: $F(1,23)=5.8, p<0.03$ ).

\section{INSERT FIGURE 1 ABOUT HERE}

The results of the foregoing analyses indicate that this experiment replicated, using an RT rather than accuracy measure of imitation, previous studies reporting that imitation of MF actions is superior to imitation of ML actions under blocked 
conditions, but this advantage is reduced or abolished under mixed conditions. To investigate the source of this interaction effect, the data from the mixed condition were subjected to further analysis. The first of these further analyses examined RTs to MF and ML action items as a function of whether each was immediately preceded by a MF or a ML item. The results, shown in the columns in Figure 2, were subjected to ANOVA in which the action type of the present trial (MF versus ML) and the action type of the previous trial (MF versus $M L)$ were within-subjects variables. A main effect of action type in the preceding trial $(F(1,23)=4.9, p<0.04)$ indicated that responding was slower when the item was preceded by a ML rather than a MF trial. No other main effects or interactions were significant. This effect of action type in the preceding trial could not have been due to a speed-accuracy trade-off because inspection of the similarity data (see Figure 2, lines) indicated that imitation was not only faster, but also more accurate, when the preceding trial involved a MF rather than a ML item. This effect was significant using a one-tailed test $(\mathrm{F}(1,23)=3.0, \mathrm{p}<$ $0.05)$.

\section{INSERT FIGURE 2 ABOUT HERE}

In the final analysis, the RT data from the mixed condition were divided into 16 successive bins of 10 trials each (Figure 3, columns), and subjected to ANOVA in which bin (1-16) and action type (MF versus ML) were within-subjects variables. (The data from two participants had to be excluded from this analysis because they did not contribute data to every one of the 32 cells of the analysis.) This analysis, and a parallel analysis applied to the similarity data (Figure 3, lines), did not produce any significant effects or interactions (both Fs $<1.2$ ). Thus, there was no evidence of a 
greater difference between MF than ML trials in early mixed trials compared with later mixed trials.

INSERT FIGURE 3 ABOUT HERE

\section{DISCUSSION}

The present study sought to replicate a previously reported effect of block composition on the imitation of MF and ML actions (Tessari \& Rumiati 2004), and investigated the roles of stimulus-driven and strategic processes in selecting between direct visuospatial, and indirect semantic, routes to imitation.

Replicating the previously reported effect, we found superior imitation of MF actions, relative to ML actions, under blocked but not under mixed conditions. The fact that this pattern of results was obtained with an RT measure of imitative performance, as well as with an accuracy measure, as in previous studies, suggests that the effect is robust, and therefore that its investigation is likely to provide important insights into the processes mediating imitation. Compared with typical measures of the accuracy of imitation of complex actions, RT measures are sensitive, objective and relatively easy to apply. Therefore, by showing that Tessari and Rumiati's effect can be obtained using an RT measure, the present study provides a useful method for future research.

The strategic selection hypothesis suggests that imitation of MF actions is superior to imitation of ML actions under blocked conditions because MF actions can be imitated 
via an indirect semantic route, which places less demand on working memory than the direct visuospatial route that must be used for ML actions. It further suggests that the advantage of MF actions disappears in mixed conditions because, after discovering that switching between direct and indirect routes is inefficient, participants strategically select the direct route for use with both MF and ML actions. In contrast, the stimulus selection hypothesis suggests that route selection is stimulus-driven, and therefore that, in both blocked and mixed conditions, ML actions are imitated via the direct route, and MF actions are imitated via the indirect route. Like the strategic selection hypothesis, it assumes that imitation of MF actions is superior to that of ML actions under blocked conditions because the semantic route places less demand on working memory. However, the stimulus selection view suggests that the advantage of MF over ML items is lost under mixed conditions because processing of ML items via the direct route depletes working memory resources, and thereby has a detrimental effect on performance in any subsequent trial. Under blocked conditions, all of the trials that incur this carry-over cost involve ML actions, but under mixed conditions, where MF and ML items are presented in random order, $50 \%$ of the trials that incur this cost are MF items.

As predicted by the stimulus selection hypothesis, the present study found that, in the mixed condition, actions were imitated faster when they followed a MF, rather than ML, action. This finding is less consistent with the strategic selection hypothesis; if MF and ML actions are both imitated via the direct route, they should place equal demands on working memory, and there should be no effect of the preceding action type on imitation of the present action. We also failed to find any evidence of strategic change in the course of testing in the mixed condition; the difference between RTs for MF and ML 
items did not change systematically throughout the mixed blocks. This is consistent with the stimulus selection hypothesis, which takes action stimulus types to determine the route of imitation, and these do not change systematically during the block. It is less consistent with the strategic selection hypothesis, which suggests that participants use the indirect route to imitate MF actions at the beginning of the mixed condition, and therefore perform better on MF than on ML trials at this stage, and that participants subsequently adopt the direct route for both MF and ML items, resulting in equivalent performance for the two action types later in the test period (Rumiati et al. 2005).

Stimulus selection may also account for neuropsychological impairments in imitation. Testing patients with apraxia, Tessari et al. (2007) reported six patients who exhibited impaired imitation of ML actions, relative to MF actions, in blocked conditions but not in mixed conditions. These patients may have damage to working memory systems (e.g. Newsome et al., 2007), which the stimulus selection hypothesis would predict are more taxed in blocked ML, and mixed, conditions. However, the finding that two patients showed impaired imitation of MF actions, relative to ML actions, in blocked conditions but not mixed conditions, is apparently at odds with stimulus selection. If the indirect route is damaged, the stimulus selection hypothesis would predict impairments when imitating MF actions in blocked and mixed conditions. However, these two patients did show a trend towards impairments in imitation of MF, relative to ML, actions in mixed as well as blocked conditions, and it is not clear whether the impairment observed under blocked conditions was reliably greater than that observed under mixed conditions.

A recent study of automatic imitation, in which participants copy observed actions without instruction, has also highlighted the importance of stimulus properties in 
determining the activation of imitation mechanisms. Human stimuli are known to be more effective than non-biological, robotic stimuli in eliciting automatic imitation (e.g. Oztop, Franklin, Chaminade, \& Cheng, 2005), and Press, Gillmeister, \& Heyes (2006) found evidence that this effect depends on low-level features of human and robotic stimuli rather than knowledge about stimulus identity (cf. Stanley, Gowen, \& Miall, 2007). However, studies of other stimulus-response associations have found clear influences of top-down processes, suggesting that, in principle, these could also mediate activation of imitation mechanisms. For example, Waszak, Wenke, \& Brass (in press) required participants to respond to stimulus targets on the basis of shape or colour dimensions, and found that responses were slower if the distractor dimension had been paired with those responses in the instructions, even if these pairings had never been presented physically.

If stimulus features are solely responsible for selection between direct and indirect routes, one might expect the advantage of MF over ML actions to be smaller in mixed than in blocked conditions, but still present when items are mixed. Although the carry-over costs of processing ML items affect both action types equally under mixed conditions, if MF items are processed by the less demanding indirect route, imitation performance for these items might still be expected to be slightly better than for ML items. Several previous experiments show a trend in this direction (Tessari \& Rumiati, 2004, Experiments 2B and 3A; Tessari, Bosanac, \& Rumiati, 2006; Toraldo, Reverberi, \& Rumiati, 2001; De Renzi, Motti, \& Nichelli, 1980). In the present study, MF actions were imitated more accurately than ML actions in the mixed condition $(F(1,23)=21.6, \mathrm{p}<0.001)$, as well as in the blocked condition $(F(1,23)=20.0, p<0.001)$, but the size of this difference was reduced in the mixed condition $(\mathrm{F}(1,23)=5.8, \mathrm{p}<0.03)$. Thus, the absence of any effect of 
action type on RT in the mixed condition of the present experiment may have been due to a speed-accuracy trade-off.

Whether route selection is driven solely by stimulus features, or by a combination of stimulus features and strategic processing, many questions remain about the neural bases, developmental origins, and differential functions of the direct and indirect routes. Using PET, Decety and colleagues (1997) found that MF actions are imitated via left frontal and temporal regions, and that ML actions are imitated via right parietal-occipital areas. If, as the stimulus selection hypothesis suggests, MF actions are consistently processed via the indirect route and ML items via the direct route, this study provides information about the neural mechanisms on which the two routes depend.

Regarding the developmental origins of the two routes, the associative sequence learning model (ASL) suggests that experience plays a crucial role (e.g. Heyes, 2001; Heyes \& Ray, 2000). According to this model, the direct route acquires its capacity to match visual with motor representations of action components from experience in which such components were simultaneously observed and executed (e.g. Heyes, Bird, Johnson, \& Haggard, 2005; Catmur, Walsh, \& Heyes, 2007; Press, Gillmeister, \& Heyes, 2007; Vogt \& Thomaschke, 2007). In contrast, the indirect route develops through acquired equivalence (Hall, 1991); experience in which a 'bridging stimulus', such as the sound of a word, is paired on some occasions with observation of an action component, and on other occasions with its execution. This experience-based account is consistent with the finding that ML actions are imitated more accurately following practice with the ML items (Tessari, Bosanac, \& Rumiati, 2006). However, it raises important questions about the precise nature of the functional specialisation of the two routes to imitation. For 
example, is the indirect route specialised for MF actions per se, or for actions that are highly familiar, or highly 'nameable' as wholes? These questions are priorities for future research.

In conclusion: The present study confirmed, using an RT measure, that imitation of MF actions is superior to that of ML actions when the two action types are presented in blocks, and that this difference is smaller or absent when they are mixed. This provides support for the view that there are two neurologically and functionally distinct routes to imitation, a direct visuospatial route and an indirect semantic route. However, the results of the present study cast doubt on the assumption that the direct and indirect routes can be strategically deployed; they suggest that these routes are automatically activated by properties of the action stimulus. 


\section{ACKNOWLEDGEMENTS}

This research was supported by the Economic and Social Research Council (ESRC) research centre for Economic Learning and Social Evolution. We are grateful to Geoffrey Bird for comments on an earlier version of the manuscript, to Richard Cook for scoring the videos, to Caitlin Orsini for help with piloting, and to Alessia Tessari and Raffaella Rumiati for allowing us to use their stimulus set, and for their advice and encouragement. 


\section{REFERENCES}

Catmur C, Walsh V, Heyes C (2007). Sensorimotor learning configures the human mirror system. Curr Biol 17: 1527-1531 (DOI: 10.1016/j.cub.2007.08.006).

Decety J, Grèzes J, Costes N, Perani D, Jeannerod M, Procyk E, Grassi F, Fazio F (1997). Brain activity during observation of actions. Influence of action content and subject's strategy. Brain 120: 1763-1777 (DOI: 10.1093/brain/120.10.1763).

De Renzi E, Motti F, Nichelli P (1980). Imitating gestures: A quantitative approach to ideomotor apraxia. Arch Neurol 37: 6-10.

Hall G (1991). Perceptual and associative learning. Oxford, England: Clarendon Press.

Heyes CM, Bird G, Johnson H, Haggard P (2005). Experience modulates automatic imitation. Brain Res Cogn Brain Res 22: 233-240 (DOI: 10.1016/j.cogbrainres.2004.09.009).

Heyes CM (2001). Causes and consequences of imitation. Trends Cogn Sci 5: 253-261 (DOI: 10.1016/S1364-6613(00)01661-2).

Heyes CM, Ray E (2000). What is the significance of imitation in animals? Adv Stud Behav 29: 215-245.

Newsome, M.R., Scheibel, R.S., Steinberg, J.L., Troyanskaya, M., Sharma, R.G., Sharma, R.G., Rauch, R.A., Li, X., \& Levin, H.S. (2007). Working memory brain activation following severe traumatic brain injury. Cortex 43: 95-111. 
Oztop E, Franklin DW, Chaminade T, Cheng G (2005). Human-humanoid interaction: Is a humanoid robot perceived as a human? Int J Hum Robot 2: 537-559.

Press C, Gillmeister H, Heyes C (2006). Bottom-up, not top-down, modulation of imitation by human and robotic models. Eur J Neurosci 24: 2415-2419 (DOI: 10.1111/j.1460-9568.2006.05115.x).

Press C, Gillmeister H, Heyes C (2007). Sensorimotor experience enhances automatic imitation of robotic action. Proc Biol Sci 274: 2639-2644 (DOI: 10.1098/rspb.2007.0774).

Rothi LJG, Ochipa C, Heilman KM (1991). A cognitive neuropsychological model of limb praxis. Cogn Neuropsychol 8: 443-458 (DOI: 10.1080/02643299108253382).

Rumiati RI, Tessari A (2002). Imitation of novel and well-known actions: The role of short-term memory. Exp Brain Res 142: 425-433 (DOI: 10.1007/s00221-0010956-x).

Rumiati RI, Weiss PH, Tessari A, Assmus A, Zilles K, Herzog H, Fink GR (2005). Common and differential neural mechanisms supporting imitation of meaningful and meaningless actions. J Cogn Neurosci 17: 1420-1431 (DOI: 10.1162/0898929054985374).

Stanley, J., Gowen, E., \& Miall, C. (2007). Effects of agency on movement interference during observation of a moving dot stimulus. J Exp Psychol Human Percept Perform 33: 915-926 (DOI: 10.1037/0096-1523.33.4.915). 
Tessari A, Bosanac D, Rumiati RI (2006). Effect of learning on imitation of new actions: Implications for a memory model. Exp Brain Res 173: 507-513 (DOI: 10.1007/s00221-006-0395-9).

Tessari A, Canessa N, Ukmar M, Rumiati RI (2007). Neuropsychological evidence for a strategic control of multiple routes in imitation. Brain 130: 1111-1126 (DOI: 10.1093/brain/awm003).

Tessari A, Rumiati RI (2004). The strategic control of multiple routes in imitation of actions. J Exp Psychol Hum Percept Perform 30: 1107-1116.

Toraldo, A., Reverberi, C., \& Rumiati, R.I. (2001). Critical dimensions affecting imitation performance of patients with ideomotor apraxia. Cortex 37: 737740 .

Vogt, S., \& Thomaschke, R. (2007). From visuo-motor interactions to imitation learning: Behavioural and brain imaging studies. J Sports Sci 25: 497-517 (DOI: 10.1080/02640410600946779).

Waszak, F., Wenke, D., \& Brass, M. (in press). Cross-talk of instructed and applied arbitrary visuomotor mappings. Acta Psychologica (DOI: 10.1016/j.actpsy.2006.12.005). 


\section{APPENDIX A}

MF actions:

1. To clean with a cloth

2. To comb

3. To paint (a wall)

4. To iron

5. To shave

6. To drink

7. To eat with a fork

8. To put on lipstick

9. To pour with a bottle

10. To brush ones own teeth

11. To stir

12. To hammer

13. To play tennis

14. To write

15. To strike a match

16. To saw

17. To cut with a knife

18. To screw in a lightbulb

19. To use a key

20. To smoke 
ML actions:

1. To clean with a cloth: cleaning performed at $90^{\circ}$ from where it is normally performed

2. To comb: combing action performed on the face from top to bottom

3. To paint: painting action performed along the main axis of body (from chest to hip)

4. To iron: ironing movement performed diagonally in front of the body

5. To shave: shaving movement performed on the chest, from bottom to top

6. To drink: drinking movement performed with the hand moving to the forehead instead of the mouth

7. To eat: eating movement performed with the hand moving to the shoulder instead of the mouth

8. To put on lipstick: drawing a circle on the chest

9. To pour: pouring movement done in reverse

10. To brush teeth: brushing movement on the shoulder

11. To stir: stirring movement performed horizontally on the left side, away from the body

12. To hammer: hammering movement performed with the hand $90^{\circ}$ from the body midline

13. To play tennis: reverse swing movement, starting from the upper-left shoulder

14. To write: writing movement above the head

15. To strike a match: striking movement along the left lower arm

16. To saw: sawing movement performed at $90^{\circ}$ from the normal position, across the front of the body 
17. To cut with a knife: cutting movement performed in reverse and on the right side of the body

18. To screw in a lightbulb: movement of screwing in a lightbulb performed $90^{\circ}$ in front of the body and toward the left side of the body

19. To use a key: turning a key at $90^{\circ}$ from the normal position, with the key pointing up

20. To smoke: smoking movement toward the chest instead of the mouth 


\section{Figure legends}

Figure 1. Mean RT, and similarity of executed actions to observed actions, when imitating MF (shaded bars, triangles) and ML (open bars, squares) actions, when presented in blocked and mixed conditions. Vertical bars indicate the standard error of the mean.

Figure 2. Mean RT, and similarity of executed actions to observed actions, when imitating MF and ML actions, in the mixed condition, when the preceding action was MF (shaded bars, triangles) and ML (open bars, squares). Vertical bars indicate the standard error of the mean.

Figure 3. Mean RT, and similarity of executed actions to observed actions, when imitating MF (shaded bars, triangles) and ML (open bars, squares) actions, at 16 successive bins during the mixed condition. Vertical bars indicate the standard error of the mean. 